\title{
MICROMACHINED CONDENSER MICROPHONE FOR HEARING AID USE
}

\author{
David Schafer, Steven Shoaf and Peter Loeppert \\ Knowles Electronics IC Group \\ Rolling Meadows, IL 60008
}

\begin{abstract}
An integrated-circuit/micromachining process, mechanical design and acoustic package have been developed which yield a condenser microphone occupying less than half the volume of earlier hearing-aid microphones. Fabrication combines a low-voltage CMOS process with surface and bulk micromachining to produce a singlechip device incorporating diaphragm, backplate, buffer amplifier and backplate bias supply, all operating from a $1.3 \mathrm{~V}$ supply (single hearing aid battery). Nominal characteristics of parts being fabricated currently are: sensitivity -40 to $-37 \mathrm{dBV} / \mathrm{Pa}$ at $1 \mathrm{KHz}$, low-frequency rolloff $150 \mathrm{~Hz}$ or below, first resonance (peak in sensitivity) at 15 to $17 \mathrm{KHz}$ and A-weighted input-referred noise 28 to $32 \mathrm{~dB}$ SPL. A lumped-element equivalent circuit model accounts for sensitivity, frequency response and thermal-mechanical noise sources in the device.
\end{abstract}

\section{INTRODUCTION}

Micromachining technology is both technologically and economically attractive for manufacture of further-miniaturized hearing-aid microphones for use in new systems such as completely in the canal (CIC) instruments. However, due to the high performance standards applied to hearing-aid microphones, careful optimization of design and materials is needed in order to achieve a micromachined device acceptable for this use. In this paper we describe a particular choice of materials, process and design, based on integrated circuit and micromachining technology, which for the first time to our knowledge has largely achieved this goal. The external appearance of the device is shown in Figure 1. The volume of this part is approximately half that of earlier hearing-aid microphones.

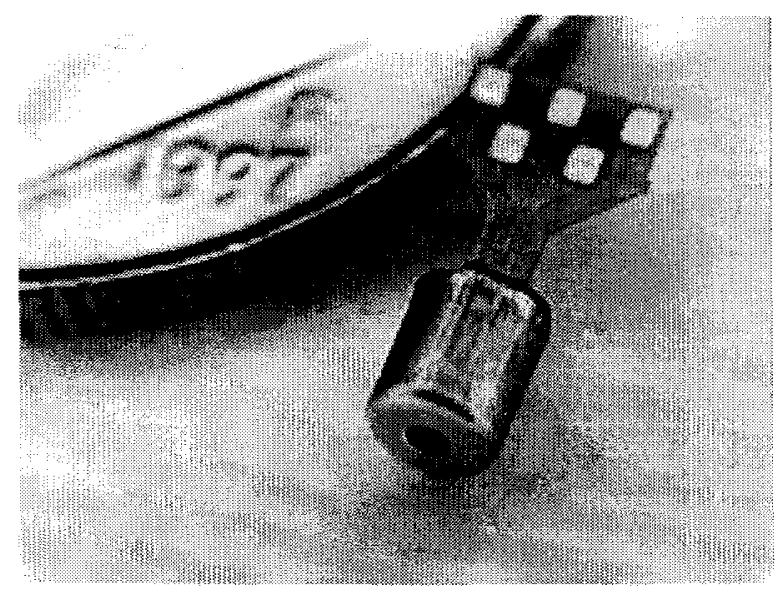

Figure 1. Assembled microphone. The coin is a dime.

The major characteristics of interest are sensitivity, input-referred noise (most simply quoted as an A-weighted rms value to correspond to the frequency range of human hearing) and frequency response. The open circuit sensitivity (volts per unit pressure) of a condenser microphone is given by

$$
\text { sensitivity }=\left(\frac{V_{b}}{C_{m}}\right)\left(\frac{d C_{m}}{d p}\right)
$$

where $V_{b}$ is the voltage applied across the diaphragm / backplate, $C_{m}$ is the capacitance of the diaphragm / backplate (which we refer to as the "motor"), and $p$ is the acoustic pressure. If for purposes of discussing scaling issues we temporarily treat the diaphragm as a piston, spaced a distance $s$ from the backplate, this expression becomes

$$
\text { sensitivity }=\left(\frac{V_{b}}{s}\right)\left(\frac{d s}{d p}\right)
$$

Electret hearing-aid microphones use stored charge to provide backplate voltages of several hundred volts. If an active circuit method is instead used to generate $V_{b}$, bias values are limited to $10-20 \mathrm{~V}$ due to the low value of the supply voltage $(1.3 \mathrm{~V})$.

In order to achieve comparable sensitivity, a non-electret microphone must have a value of $(1 / s)(d s / d p)$ roughly ten times higher than that of an electret microphone. As shown in published examples of micromachined devices [1-6], this requirement tends to drive designs toward thin diaphragms (a few microns ) and/or small motor air gap dimensions (also a few microns). For a diaphragm diameter less than $1 \mathrm{~mm}$ as discussed here, the diaphragm thickness must be near 1 micron for adequate compliance. This is compatible with integrated circuit and micromachining processes, although residual membrane stress in the finished diaphragm must be kept very low (typically $1 \mathrm{MPa}$ or less) to preserve the compliance gained. Also, the need for control of the motor air gap means that any distortion of the diaphragm due to residual stress or stress gradients must be small compared to a few microns.

Once micron-sized motor air gaps are adopted, squeeze-film damping of the diaphragm motion due to viscosity of the air in the gap becomes significant $[\mathbf{1 , 2 , 7 , 8}]$, first of all because of its contribution to the thermal-mechanical noise of the device [9] and secondarily because of its effect on frequency response.

\section{DESIGN}

In the device described here, high compliance is achieved in a small space by arranging the diaphragm structure in a "mushroom" form, specifically a disc which is anchored at the center and free to bend at the edges. Due to the mechanical boundary conditions set up in this arrangement, this structure has approximately five times the linear compliance of an edge-supported diaphragm of the same diameter. For the diaphragm material used here $(0.75$ micron PECVD nitride), the compliance is higher than necessary. Corrugations are added to the diaphragm to bring it to the desired compliance. 
In the mushroom configuration, as-deposited stress in the diaphragm material is relieved except for the area around the anchor. Finite element simulation studies indicate that this area of unrelieved stress has very little impact on the diaphragm position.

Since the greatest compliance in this structure occurs at the diaphragm edge, the working area of the motor is located there. As shown in Figure 2, the backplate is a tensioned thin film of 1.1 micron PECVD/LPCVD silicon nitride which is largely cut away in regions near the center of the device. The working area is an annular region at the diaphragm edge, of width equal to about one-fourth of the diaphragm radius. In this working area, the backplate is further perforated to form a web-like structure of low acoustic resistance.

A portion of the diaphragm edge, of minimal width and airgap, overlaps the backplate to form a controlled-resistance pressure relief path.

The source capacitance of the motor is about $0.2 \mathrm{pf}$. The onchip amplifier is an MOS follower circuit with an input capacitance of $0.2 \mathrm{pf}$ and an A-weighted output noise level of approximately -108 $\mathrm{dBV} .12 \mathrm{~V}$ bias is provided to the backplate by an on-chip ring oscillator and charge pump.

\section{FABRICATION PROCESS}

The device is fabricated using a low-voltage CMOS process sequence followed by additional process steps which build up the transducer structure. The PECVD silicon nitride passivation layer of the CMOS process, tensioned with an LPCVD nitride layer preserved from a LOCOS step of the CMOS process, is used to form the backplate web. The net tensile stress of this two-layer film stiffens the web to the point that its compliance is negligible compared to that of the diaphragm. The backplate is metallized with chrome.

Two sacrificial layers are deposited and patterned next. The first, 3 microns thick, is used to form corrugated relief in the surface which is eventually replicated in the diaphragm. The second, 1 micron thick, is a blanket layer which defines the regions where the diaphragm is to be anchored. In the motor working area the air gap is 4 microns. while at the diaphragm edge (pressure relief path) the gap is 1 micron.

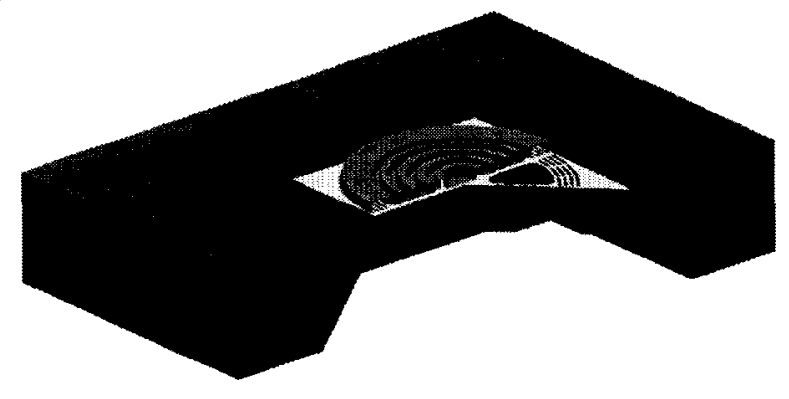

Figure 2. Finished micromachined die, cut away to reveal the configuration of the thin-film diaphragm and backplate, as well the front and back holes.

A second layer of PECVD nitride ( 0.75 micron thick) is deposited and patterned for use as the diaphragm. The diaphragm is also metallized with chrome. Finally TiW/Au is deposited and patterned for use in leadout and bond pads.

The finished wafers are micromachined in $\mathrm{KOH}$. Etching occurs on both the front and back surfaces, creating holes which meet halfway through the wafer. The layout of the passivation web on the front causes four individual holes to be formed on the front side of the wafer, with "ribs" of unetched silicon between them. A cutaway view of the resulting structure is shown in Figure 2.

A plan view of a microphone die, $2 \times 2 \mathrm{~mm}$ in size, is shown in Figure 3. The diaphragm is $0.8 \mathrm{~mm}$ in diameter. There is considerable unused silicon area, which allows more circuitry to be added or the die size to be reduced.

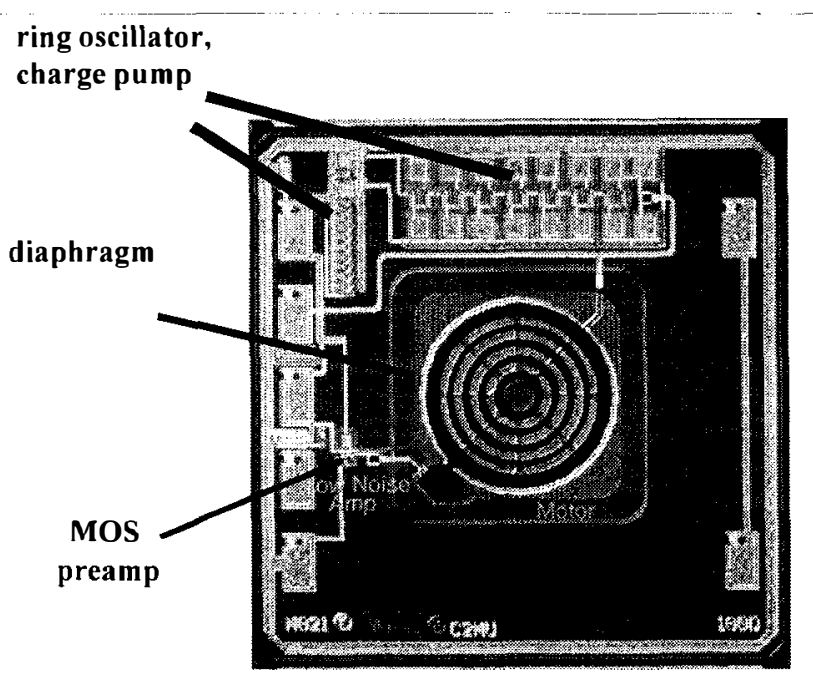

Figure 3. Plan view of microphone die

\section{PACKAGING}

The device is assembled into a cylindrical package, shown in Figure 4. The micromachined die is first $\mathrm{TAB}$ bonded to a copper/polyimide flexible lead set, then mounted in an injectionmolded plastic "cradle", next held in place in the stainless steel case using a second plastic insert, and finally sealed in place with heatcuring encapsulant. The cradle incorporates the acoustic back cavity required for the diaphragm, and the acoustic path formed by both plastic parts creates a light-blocking port. The case diameter is $0.090^{\prime \prime}$ and the case length $0.105 "$.

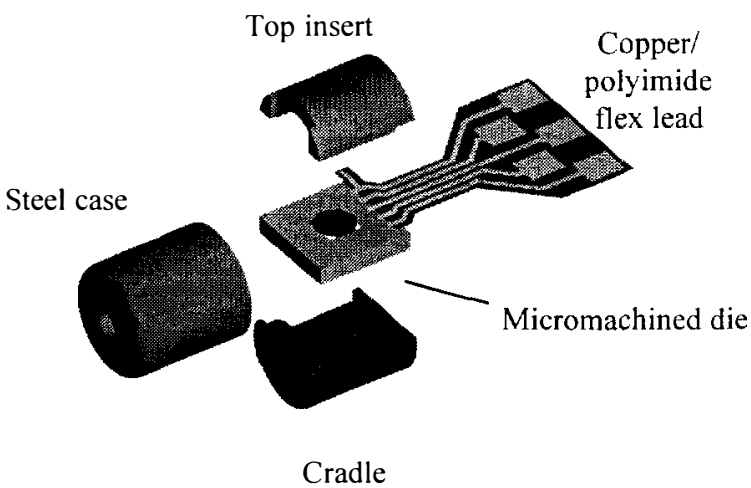

Figure 4. Exploded view of packaging arrangement 


\section{DEVICE PERFORMANCE}

Nominal performance characteristics of parts being fabricated currently are: sensitivity -37 to $-40 \mathrm{dBV} / \mathrm{Pa}$ at $1 \mathrm{KHz}$, low-frequency rolloff $150 \mathrm{~Hz}$ or less, first resonance (peak in sensitivity) at 15 to 17 $\mathrm{KHz}$, and A-weighted input-referred noise 28 to $32 \mathrm{~dB}$ SPL. The frequency response is shown in Figure 5. In an alternate design, with a smaller, more highly damped motor and lower-inertance port, usable response extends out to $30 \mathrm{KHz}$ which makes the device useful for ultrasonic sensing applications.

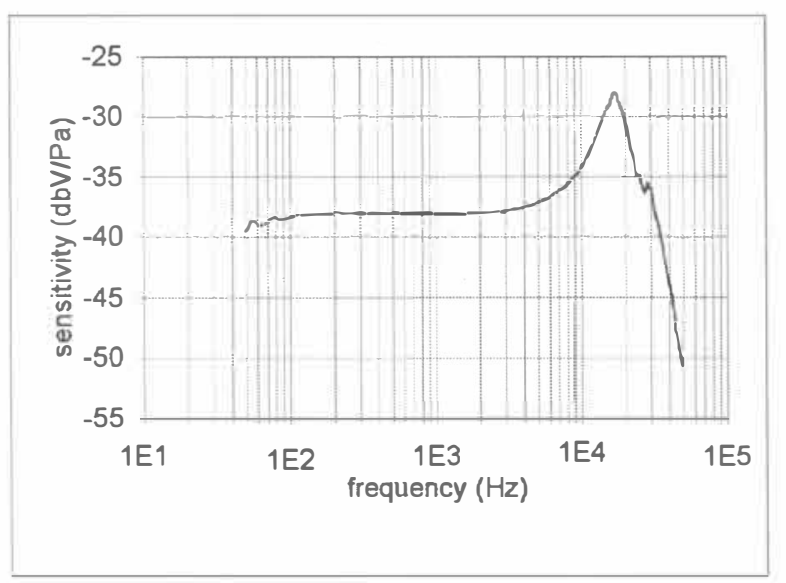

Figure 5. Frequency response

\section{DEVICE MODELING}

A lumped-element electrical-acoustical equivalent circuit model of the device, shown in Figure 6, is useful for accounting for sensitivity, frequency response and noise. In the left half of the equivalent circuit, current represents volume displacement rate $u$ and electric potential represents acoustic pressure $p$. The port, front volume, back cavity, motor inertance and motor damping are represented using two-terminal passive elements.

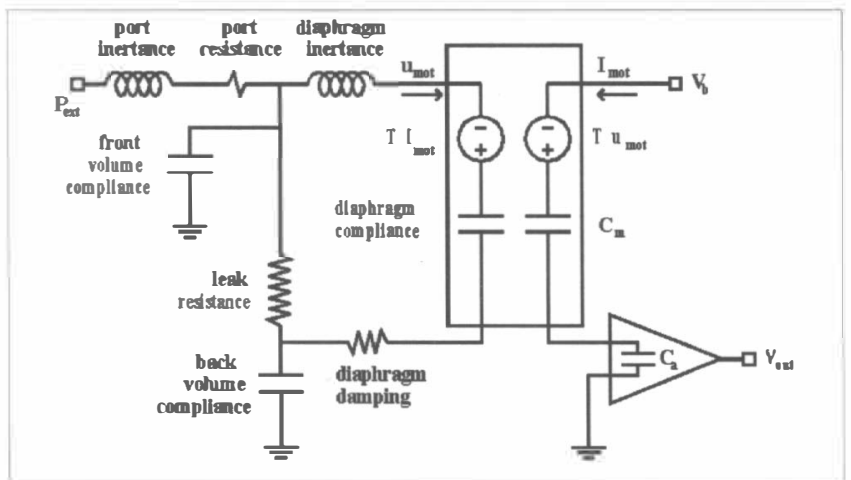

Figure 6.Lumped-element equivalent circuit model of microphone

The transducer characteristics of the motor at angular frequency $\omega$ are represented as a four-terminal element with two passive elements (volume compliance, electrical capacitance of the motor) and two dependent sources which represent the effect of the motor volume displacement rate $u$ on the net motorvoltage $v$, and the effect of the motor current $i$ on the net diaphragm pressure load $p$ :

$v=T \times u ; p=T \times i ; T=\left(\frac{i}{\omega}\right)\left(\frac{V_{b}}{C_{m}}\right)\left(\frac{d C_{m}}{d(\text { volume })}\right)$
The buffer amplifier and its input capacitance $C_{a}$ are represented in the right half of the figure.

The values of several elements in the equivalent circuit model may be obtained from an ac measurement in which a small ac drive signal $v_{b}$ is applied to the backplate terminal (overriding the output of the on-chip charge pump which has very little current capacity) and measuring the amplitude and phase of the ac gain $v_{\text {our }} / v_{b}$ where $v_{\text {out }}$ is the output signal. The ac gain depends on the ratio of the net electrical impedance of the motor to the capacitive input impedance of the buffer amplifier. At zero dc backplate bias the transducer is inactive and the ac gain depends just on $C_{m} / C_{a}$. Since $C_{a}$ is known from other wafer-level measurements then the value of $C_{m}$ for the model can be determined and consistency with the nominal air gap and motor area checked. At non-zero dc backplate bias ( $T$ not zero), the ac gain measurement couples to the mechanical side of the system. By sweeping the drive frequency through the appropriate range, we can identify mechanical resonances of the device from the corresponding frequency dependence in the ac gain.

To study the dynamics of the motor alone, we have done ac gain measurements on micromachined, unpackaged die as shown in Figure 7. In this case the diaphragm is the only compliance present.

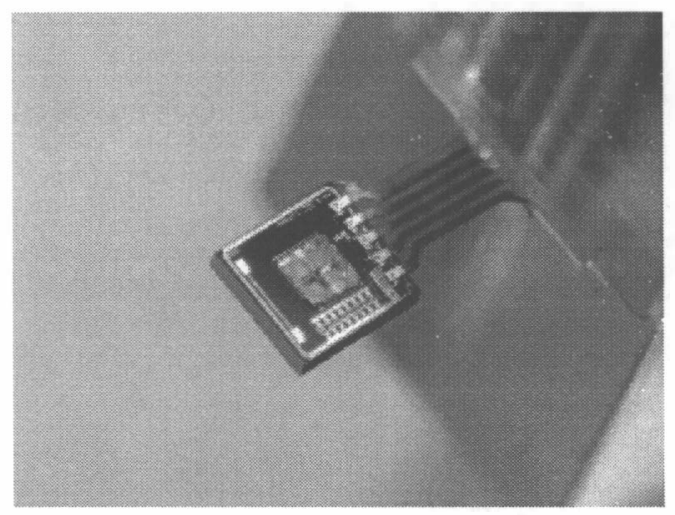

Figure 7. ac gain measurement on a micromachined, unpackaged die, used to study motor dynamics.

As the frequency is swept through the diaphragm resonance, the real part of the ac gain shows a relatively small shift (only a few percent), but the imaginary part shows a dip whose center frequency and width can easily be measured.

Figure 8 shows the out-of-phase component (quadrature signal from a lockin amplifier) of the ac gain signal vs. frequency for a particular part (\#1597) under different ambient conditions. Each plotted curve is the result of subtracting a zero-backplate-bias (baseline) sweep from a second sweep at $12 \mathrm{~V}$ bias.

Vacuum measurement of the resonance provides the clearest indication of the diaphragm characteristics alone since the inertance and damping effects of air are then removed. Under these conditions the resonance is relatively narrow. The resonant frequency at 29.4 $\mathrm{KHz}$ under rough vacuum in Figure 8 agrees with the frequency of a "piston-like" vibrational mode of the diaphragm seen at $28.9 \mathrm{KHz}$ in finite-element analysis simulations. This tends to confirm the accuracy of the finite-element analysis for other calculations such as the quasi-static compliance of the diaphragm. 


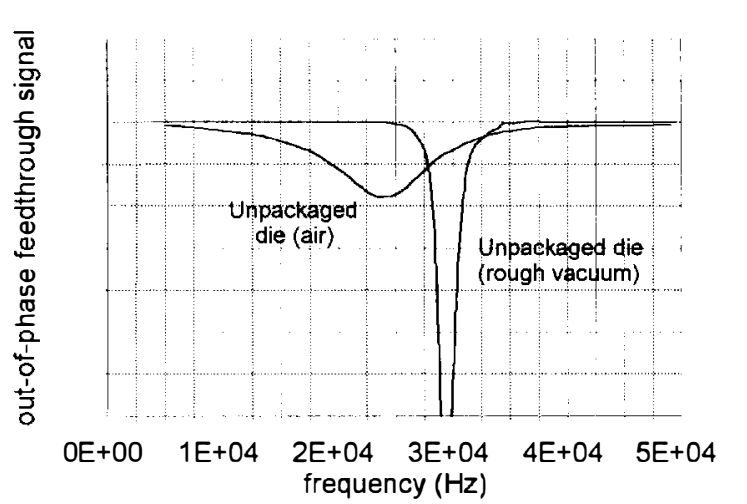

Figure 8. Mechanical resonances seen in ac gain spectrum.

When the ac gain spectrum is re-measured in air the resonance shifts downward to $24.4 \mathrm{KHz}$ and the width increases to $9.7 \mathrm{KHz}$. The resonance width change is accounted for to within about $25 \%$ by a manual estimate of the damping effects of air viscosity in the motor gap assuming laminar flow with a parabolic velocity profile. Thus the damping seen in the unpackaged die as appears to be internal to the motor and thus able to be carried over without modification to the model of the packaged device. The estimated inertance of air in the motor gap, on the other hand, does not account for the observed frequency shift, which appears to come from the mass loading effects of ambient air and the inertance of the holes in the die.

When the part of Figure 8 is mounted on a variable-volume test cavity set at $2 \mathrm{~mm}^{3}$ (equivalent to the package "cradle"), the resonance moves back up to about $26 \mathrm{KHz}$. In fully packaged parts the resonance finally moves back down to 15 to $17 \mathrm{KHz}$ as seen as the peak in the sensitivity (Figure 5). Reproducing these successive shifts in the ac gain of the equivalent circuit determines the port inertance and back volume compliance values of the model.

\section{NOISE ANALYSIS}

The output voltage noise spectrum of the part discussed above (\#1597) is shown in Figure 9. The part is still on the test cavity, now set at $2.9 \mathrm{~mm}^{3}$ volume to bring the resonance down somewhat.

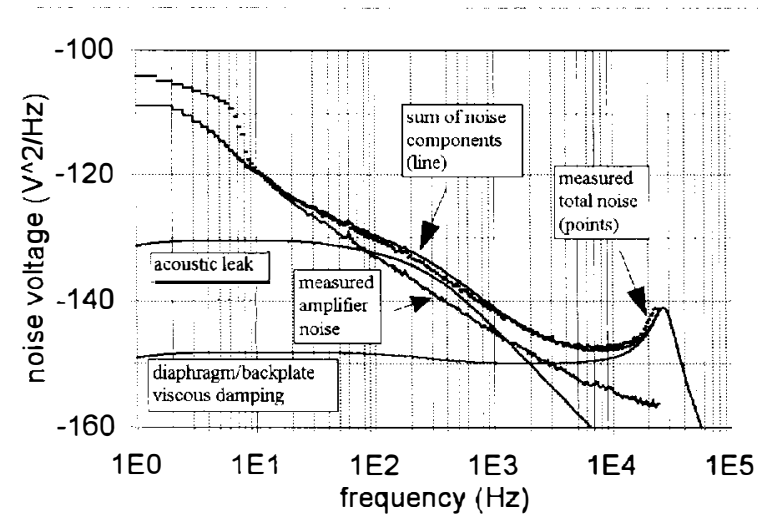

Figure 9. Noise spectrum of the part shown in Fig. 8, mounted on the test cavity, set at $2.9 \mathrm{~mm}^{3}$ volume.

Noise spectra at $V_{b}=0$ and $V_{b}=12 \mathrm{~V}$ are shown. At $V_{h}=0$ the transducer is inactive and only electronic noise sources appear, mainly the $1 / \mathrm{f}$ noise of the amplifier. At $V_{h}=12 \mathrm{~V}$, two additional features appear: a 3-4 dB increase over a broad range from $100 \mathrm{~Hz}$ to $5 \mathrm{KHz}$ and a sharp rise above $10 \mathrm{KHz}$.

Thermal noise of the acoustic resistances in the equivalent circuit model account for these added noise features quite well. Two additional curves in Figure 9 show the model-predicted contributions to the output voltage noise from the motor damping resistance and the leak resistance, each taken as a pressure noise source of spectral density $P^{2}=4 k T R$, where $R$ is the respective acoustic resistance. Added to the amplifier noise, the total is accounted for to within 1 or $2 \mathrm{~dB}$. On an A-weighted basis, the buffer amplifier, relief path resistance and diaphragm damping each contribute about one-third to the total input-referred noise, which is $32 \mathrm{~dB}$ SPL in this example.

\section{ACKNOWLEDGEMENTS}

The authors would like to acknowledge the contributions of the IC fabrication group at KEICG, Jay Cech, Engineering Manager, and Ian Arnott, Director. We would also like to acknowledge Karen Crow, Irene Gomez and Debora Nicholson for implementation of the assembly process and for acoustic and electrical measurements.

\section{REFERENCES}

1. J. Berqvist, F. Rudolf, J. Maisano, F. Parodi and M. Rossi, "A Silicon Condenser Microphone with a Highly Perforated Backplate", Technical Digest of the 6th International Conference on Solid-State Sensors and Actuators (Transducers '91), San Francisco, CA, USA, 6/24-28/91, pp.266-269.

2. J. Bernstein, "A Micromachined Silicon Condenser Microphone with On-Chip Amplifier", Technical Digest of the 1996 Solid-State Sensor and Actuator Workshop, Hilton Head Isl., SC, 6/3-6/96, Transducer Research Foundation, Cleveland (1996), pp. 239 - 243.

3. P. Scheeper, W. Olthuis and P. Bergveld, "Improvement of the Performance of Microphones with a Silicon Nitride Diaphragm and Backplate", Sensors and Actuators A, 40, 179-186 (1994).

4. Y. Ning, A. Mitchell and R. Tait, "Fabrication of a Silicon Micromachined Capacitive Microphone Using a Dry-Etch Process", Sensors and Actuators A, 53, 237-242 (1996).

5. Q. Zou, Z. Li and L. Liu, "Design and Fabrication of Silicon Condenser Microphone Using Corrugated Diaphragm Technique", Journal of Microelectromechanical Systems, 5, 94 (1996).

6. M. Pedersen, W. Olthuis and P. Bergveld, "A Silicon Condenser Microphone with Polyimide Diaphragm and Backplate", Sensors and Actuators A, 63, 97-104 (1997).

7. Z. Skvor, "On the Acoustical Resistance Due to Viscous Losses in the Air Gap of Electrostatic Transducers", Acustica, 19, 295-299 (1967/68).

8. G. Plantier and M. Bruneau, "Heat Conduction Effects on the Acoustic Response of a Membrane Separated by a Very Thin Air Film from a Backing Electrode", J. Acoustique, 3, 243-250 (1990).

9. T. Gabrielson, "Mechanical-Thermal Noise in Micromachined Acoustic and Vibration Sensors", IEEE Trans. Elect. Devices, 40, 903-909 (1993). 